\title{
Lake-Stream Divergence in Stickleback Life History: A Plastic Response to Trophic Niche Differentiation?
}

\author{
Dario Moser $^{1} \cdot$ Benjamin Kueng $^{1} \cdot$ Daniel Berner $^{1}$
}

Received: 4 March 2015/ Accepted: 16 May 2015/Published online: 26 May 2015

(C) Springer Science+Business Media New York 2015

\begin{abstract}
Speciation can be promoted by phenotypic plasticity if plasticity causes populations in ecologically different habitats to diverge in traits mediating reproductive isolation. Although this pathway can establish reproductive barriers immediately and without genetic divergence, it remains poorly investigated. In threespine stickleback fish, divergence in body size between populations represents a potent source of reproductive isolation because body size often influences reproductive behavior. However, the relative contribution of phenotypic plasticity and genetically based divergence to stickleback body size evolution has not been explored. We here do so by using populations residing contiguously in Lake Constance (Central Europe) and its tributaries, a system where lake fish exhibit strikingly larger size and greater age at maturity than stream fish. Laboratory experiments reveal the absence of substantial genetic divergence in intrinsic growth rates and maturation size thresholds between lake and stream fish. A field transplant experiment further demonstrates that lake fish display the life history typical of stream fish when exposed to stream habitats for one year, confirming that life history divergence in this system is mainly plastic. This plasticity appears to be driven by restricted food availability in the lake relative to the stream
\end{abstract}

Electronic supplementary material The online version of this article (doi:10.1007/s11692-015-9327-6) contains supplementary material, which is available to authorized users.

Daniel Berner

daniel.berner@unibas.ch

1 Zoological Institute, University of Basel, Vesalgasse 1, Basel, Switzerland habitat. We thus propose that in this stickleback system, the exploitation of different trophic niches immediately promotes reproductive isolation via resource-based plasticity in life history.

Keywords Field transplant experiment - Gasterosteus aculeatus - Growth rate · Maturation size threshold . Phenotypic plasticity $\cdot$ Reproductive isolation

\section{Introduction}

The formation of new species is often initiated by the divergence of populations into selectively different habitats (Rice 1987; Schluter 2000; Rundle and Nosil 2005; Sobel et al. 2010; Nosil 2012). Generally, two key elements are implicitly assumed to govern this process: first, divergent selection on phenotypes between habitats drives allele frequency shifts between populations at underlying genetic loci. Second, this genetically based (i.e., heritable) divergence generates some degree of reproductive isolation between the populations, for instance through performance tradeoffs between the habitats (Hendry 2004; Nosil et al. 2005; Thibert-Plante and Hendry 2009), or divergence in reproductive behavior (Coyne and Orr 2004; Ritchie 2007; Maan and Seehausen 2011; Thibert-Plante and Gavrilets 2013). Although it is debatable how fast reproductive isolation through this pathway can emerge (Hendry et al. 2007; Gavrilets et al. 2007; Nosil 2012), selection over multiple generations is certainly needed to achieve the underlying genetic divergence-even when selection is strong and genetic variation is abundant.

However, a faster pathway to speciation can occur when the exposure to ecologically different habitats directly causes divergence between populations through phenotypic 
plasticity (i.e., not via allele frequency shifts), and this divergence drives reproductive isolation. For example, if plasticity generates adaptive population differentiation prior to dispersal, gene flow between habitats will be impeded by selection against migrants (Crispo 2008; Thibert-Plante and Hendry 2011; Fitzpatrick 2012). Similarly, plasticity might cause phenotypic divergence in traits mediating reproductive interactions, such as mating cues or phenology (Levin 2009), and thereby produce assortative mating. In all these scenarios involving phenotypic plasticity, reproductive barriers will arise within a single generation and set the stage for further divergence through allele frequency changes. Despite this potentially important role of plasticity in speciation, however, research efforts are generally directed to deciphering how genetically based trait differences between diverging populations contribute to reproductive isolation (e.g., Hatfield 1997; Hawthorne and Via 2001; Lexer et al. 2004; Rogers and Bernatchez 2006; Terai et al. 2006; Rego et al. 2007; Fuller 2008; Kitano et al. 2009; Lowry and Willis 2010; Berner et al. 2011; Streisfeld et al. 2013; Arnegard et al. 2014; Chung et al. 2014; but see Payne et al. 2000; Kozak et al. 2011; Smith et al. 2013).

In the present study, we begin an investigation of the potential role of phenotypic plasticity in speciation by using populations of threespine stickleback fish (Gasterosteus aculeatus $\mathrm{L}$.) residing in contiguous lake and stream habitats (Reimchen et al. 1985; Lavin and McPhail 1993; Hendry and Taylor 2004; Berner et al. 2009; Ravinet et al. 2013). We focus on lake-stream stickleback populations occurring in the Lake Constance basin in Central Europe (Berner et al. 2010; Lucek et al. 2010; Moser et al. 2012). In this system, stickleback exploit two distinct trophic niches: lake fish feed pelagically (i.e., in the open-water) on zooplankton during most of their life, whereas stream fish feed exclusively on benthic (bottom-dwelling) macro-invertebrates (Berner et al. 2010; Lucek et al. 2012; Moser et al. 2012). This differential habitat use is paralleled by substantial divergence in putatively neutral genetic markers over small geographic scales (Berner et al. 2010; Moser et al. 2012). Because Lake Constance stickleback invade tributaries during the breeding season, providing the opportunity for genetic exchange between lake and stream fish, the small-scale genetic structure indicates the presence of at least partial barriers to gene flow between the habitats.

The nature of these barriers is presently unknown but might be related to divergence in life history. Specifically, stickleback in the Lake Constance basin display strong lake-stream divergence in age and size at reproduction: Lake Constance fish generally start reproducing when they are 2 years old and large, while their counterparts in the tributaries typically die after reproducing at one year of age and at only half the body mass of the lake fish (Fig. 1a) (Lucek et al. 2012; Moser et al. 2012). Experimental evidence from other threespine

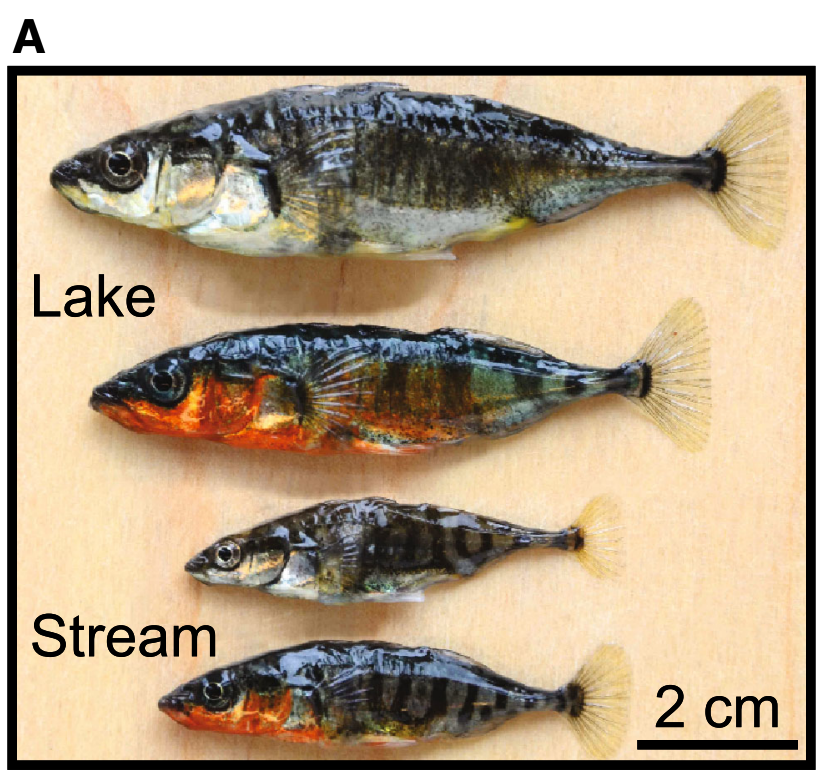

B

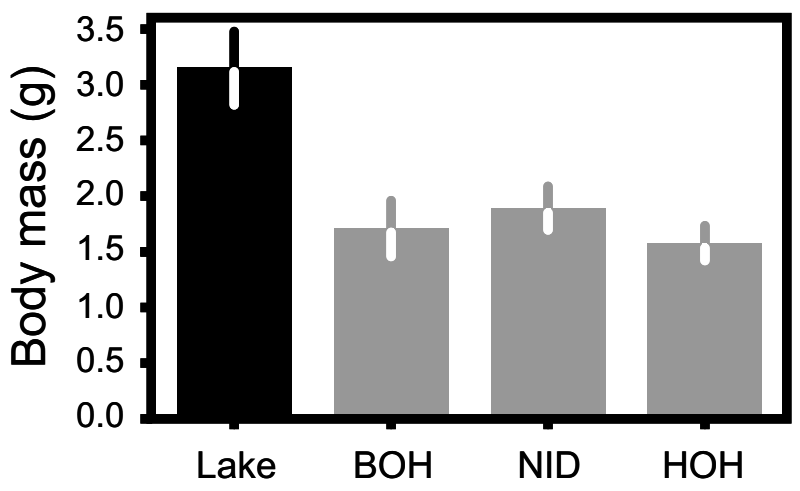

Fig. 1 a Reproductive stickleback from Lake Constance and an inlet stream (NID). Females are on top, males (in breeding dress) on the bottom. b In the wild, Lake Constance stickleback display roughly twice the body size of stickleback populations residing in tributaries (BOH, NID, HOH). Error bars are parametric $95 \%$ confidence intervals

stickleback systems suggests that this life history divergence might be an important component of reproductive isolation. The reason is that stickleback mate choice has often been found to be positively size-assortative, with both females and males mating preferably with conspecifics of matching size (Nagel and Schluter 1998; Ishikawa and Mori 2000; McKinnon et al. 2004, 2012; Albert 2005; Boughman et al. 2005; Conte and Schluter 2013; but see Raeymaekers et al. 2010). Similarly, the outcome of aggressive territorial interactions among males has been shown to be body size-dependent (Dufresne et al. 1990; Nagel and Schluter 1998). We have therefore suggested that the divergence in body size observed in the Lake Constance system might contribute to reproductive isolation between lake and stream populations (Moser et al. 2012). An important step in exploring this idea is to understand the relative contribution of adaptive genetic 
divergence versus phenotypic plasticity to life history divergence, since these alternative mechanisms determine how rapidly reproductive isolation can emerge.

We here present such an investigation based on predictions from a mechanistic model of resource allocation. In particular, it is a general feature of animal ontogeny that the transition from a primarily somatic growth phase to the reproductive stage is governed by a maturation size threshold (reviewed in Bernardo 1993; Nijhout 2003; Berner and Blanckenhorn 2007; for evidence from threespine stickleback see Craig-Bennett 1931). Based on this recognition, the divergence in life history between lake and stream stickleback might be achieved in two ways: first, lake and stream fish share a common maturation size threshold, but lake fish grow more slowly than stream fish (Fig. 2a). As a consequence, only stream stickleback reach the maturation size threshold within one year of growth and can thus respond to the photoperiodic cue (critical day length) that triggers reproduction (Craig-Bennett 1931; Baggerman 1985). Second, lake and stream fish exhibit similar growth trajectories but lake fish have a higher maturation size threshold than stream fish (Fig. 2b; see also Shimada et al. 2011). Consequently, again only stream stickleback manage to enter the reproductive stage after one year of growth. It is important to note

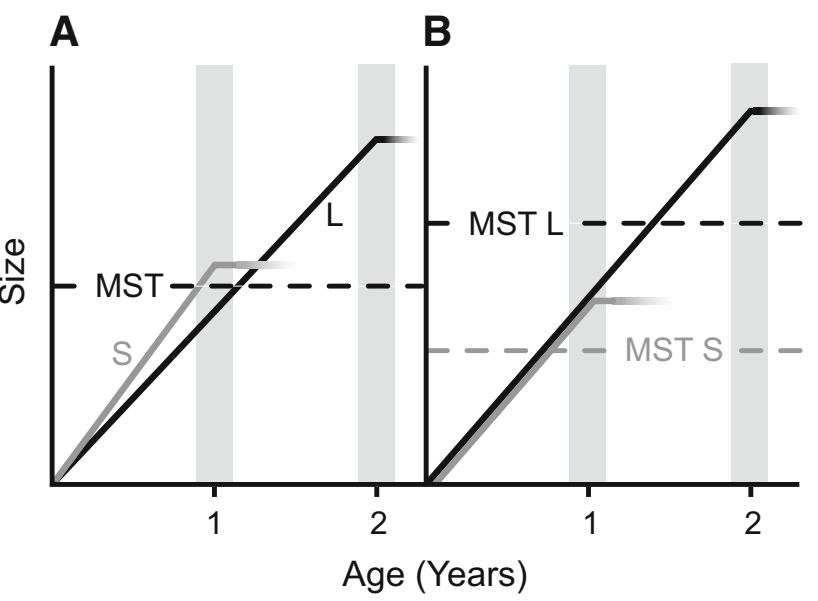

Fig. 2 Alternative models of life history divergence between lake and stream stickleback. a Lake (L) and stream (S) fish share a common maturation size threshold (MST) but differ in growth rates. Stream fish grow fast and reach critical size by one year of age. They thus respond to the first photoperiodic cue (gray vertical bar) by maturing and reproducing, followed by senescence and death. By contrast, body size in the relatively slow-growing lake fish is still below the maturation size threshold after one year, so that the first photoperiodic cue cannot trigger reproduction. Instead, lake fish continue to invest in somatic growth. After 2 years, the lake fish have grown beyond critical size (and beyond the size of reproductive stream fish) and are ready to mature. b Lake and stream fish share similar growth rates but exhibit different maturation size thresholds. A relatively low size threshold in stream fish allows them to mature after one year, whereas a higher critical size in lake fish allows reproduction after 2 years only. Both models lead to the joint divergence in age and size at maturity that these two mechanisms of life history divergence are not mutually exclusive, and that both may be influenced by genetically based divergence, phenotypic plasticity, or a combination of the two.

To shed light on these different possibilities, we use laboratory experiments examining if lake and stream populations have evolved genetically based differences in the intrinsic growth rate, and/or in the maturation size threshold. These experiments are complemented by a field transplant experiment to evaluate to what extent life history is phenotypically plastic. As we will show, these experiments together indicate a key role of plasticity in life history divergence between lake and stream stickleback, with potentially important consequences for speciation in the face of gene flow.

\section{Materials and Methods}

\section{Study Populations and Generation of Experimental Lines}

All our experiments described below were performed by using F1 individuals derived in the laboratory from stickleback caught from one Lake Constance site and from three inlet stream populations. Consistent with previous work, the lake population was sampled in Romanshorn (for geographic details see Berner et al. 2010; Moser et al. 2012). A single sample was adequate to represent Lake Constance stickleback because they are known to form a large, genetically well-mixed population (Berner et al. 2010; Moser et al. 2012). The stream populations are those from Bohlingen (BOH), Nideraach (NID), and Hohenems (HOH) (Berner et al. 2010; Moser et al. 2012). Sampling occurred with unbaited minnow traps during the breeding season (early May 2013). The collected individuals were immediately transferred to the laboratory to perform artificial crosses. All crosses were made within a window of 3 weeks, and each specimen was used for a single cross only. We generated 12 pure lake crosses and ten pure crosses for each stream population. The 84 total parental individuals and some surplus fish not used for crossing (lake: $\mathrm{N}=11$; $\mathrm{BOH}$ : $\mathrm{N}=10$; NID: $\mathrm{N}=6$; $\mathrm{HOH}: \mathrm{N}=7$ ) were killed with an overdose of Koi Med Sleep (phenoxyethanol; Fishmed, Rain, Switzerland), weighed to the nearest $0.01 \mathrm{~g}$, and stored in absolute ethanol. Following the protocol in Moser et al. (2012), we subsequently determined otolith-based age at reproduction. All these field-caught fish (grand total $\mathrm{N}=118$ ) were used to confirm that the lake-stream divergence found in Moser et al. (2012) was constant across years (i.e., samples from 2010 vs. 2013), and to compare life histories between experimental and wild fish.

Embryonic development of the F1 generation occurred in four well aerated $100 \mathrm{~L}$ tanks, with $2 \mathrm{~g} \mathrm{NaCl}$ and $80 \mu \mathrm{l}$ 
Fungol (JBL, Neuhofen, Germany) per liter to prevent fungus infection (none observed). For hatching, each clutch (family; $\mathrm{N}=42$ ) was transferred to an individual $15 \mathrm{~L}$ 'hatching tank' connected to a flow-through system. We used a rearing temperature of $16{ }^{\circ} \mathrm{C}$ and a $16: 8 \mathrm{~h}$ lightdark (LD) summer photoperiod. Hatchlings were fed live Artemia nauplii ad libitum twice a day during the first 2 weeks, and then additionally frozen copepods (Cyclops).

\section{Laboratory Experiment 1: Genetically Based Divergence in Growth Trajectories}

In our first laboratory experiment, we tracked stickleback body mass (our body size measure) over one year to investigate whether lake and stream populations have evolved different intrinsic growth trajectories. Based on the ontogenetic model above, our specific prediction was that stream fish display steeper trajectories (Fig. 2a).

This experiment involved the Lake Constance (hereafter simply 'lake') population and two stream populations (BOH and NID). Three weeks after hatching, we haphazardly selected 40 individuals from each hatching tank and divided this sample into two replicate $15 \mathrm{~L}$ tanks connected to the same flow-through system (resulting in 84 total tanks with 20 individuals each). As a resource for the other experiments described below, the remaining fish were transferred from the hatching tanks to $100 \mathrm{~L}$ 'stock tanks', pooling all families within each population. Although this precluded subsequent tracking of the source families, the number of individuals per family was relatively similar and mortality was near zero, thus ensuring a balanced contribution to the stock tanks across families. The fish used for the growth trajectory experiment and those in the stock tanks received exactly the same temperature, light, and food treatment. Specifically, the temperature was $16{ }^{\circ} \mathrm{C}$ throughout the experiment. The photoperiod was initially 16:8 h LD ('summer') but was reduced to 12:12 16 weeks post-hatch and to $8: 16$ ('winter') 22 weeks post-hatch until the end of the experiment. The winter photoperiod was used to avoid the transition to reproduction toward the end of the experiment. All fish were fed ad libitum twice a day. We provided a mix of live Artemia nauplii and frozen Cyclops during the first 9 weeks, and then a combination of frozen bloodworms (chironomid larvae), frozen Cyclops, and decapsulated Artemia eggs. This latter diet included prey taxa consumed by both limnetically foraging lake stickleback and benthically foraging stream stickleback populations in the wild (e.g., Berner et al. 2008, 2010; Moser et al. 2012).

In the course of the growth trajectory experiment, the number of individuals per tank was reduced from initially 20 down to 15 (10 weeks post-hatch), 13 ( 29 weeks), and 11 (32 weeks), eliminating individuals haphazardly. The rationale of this reduction was to avoid excessive crowding, and to maintain similar densities of individuals across the tanks (although mortality was very low). Starting 9 weeks post-hatch, ten fish per tank were chosen haphazardly, weighed to the nearest $0.01 \mathrm{~g}$, and returned to their tank. This was repeated every 5 weeks, resulting in nine rounds of measurement and a total experimental period of one year. In each round except the last, measurements were conducted within 7 days or less. The ninth round was performed in the exact order of hatching over a window of 14 days and included all remaining fish per tank (mean: 10.7).

\section{Laboratory Experiment 2: Genetically Based Divergence in Maturation Size Thresholds}

The second laboratory experiment tested the prediction derived from the ontogenetic model that lake fish have evolved an elevated maturation size threshold relative to stream fish (Fig. 2b). Our experimental strategy was to transfer stickleback from both habitats from a winter to a summer photoperiod (i.e., to provide the photoperiodic maturation cue) at body sizes well below the size of reproductive lake fish in the wild. Our expectation was that if maturation size thresholds have diverged between the habitats, lake fish would display a lower propensity to respond to the cue and become mature than stream fish.

This experiment used lake and NID stream individuals chosen haphazardly from the corresponding stock tanks and was conducted in a separate room with $16^{\circ} \mathrm{C}$ and a summer photoperiod. The room was equipped with fifty 15 L tanks. Each tank was furnished with $400 \mathrm{ml}$ of sand and fine gravel, 200 nylon threads of $5 \mathrm{~cm}$ length (both to allow males to build a nest), and a plastic plant. To start the experiment, each tank was stocked with a single individual whose body mass had been recorded. Visual contact among tanks was allowed. The fish were fed Cyclops and frozen bloodworms ad libitum twice a day. Before feeding, we inspected the reproductive status of each individual. Females were considered reproductive if they produced a ripe clutch that could be stripped. Males qualified as reproductive if they displayed breeding dress (bluish iris and orange jaw and throat) and maintained a nest. In a few cases where the latter criterion was ambiguous, we presented a gravid female to the male. If the male then displayed and entered its nest, it was considered reproductive. The sex of individuals not mature at the end of the experiment was determined by dissection.

The experiment was conducted in three rounds, starting 32,39 , and 46 weeks post-hatch, each lasting 45 days. All experimental fish had thus experienced at least 10 weeks of winter photoperiod in the stock tanks. Total sample size was 150, with 81 lake fish $(\mathrm{N}=34,20$, and 27 in the rounds 1 , 
2, and 3) and 69 NID stream fish $(\mathrm{N}=16,30,23)$. Mortality was zero, but eight fish showed low food uptake and apathy and were excluded from analysis.

\section{Field Transplant Experiment: Life History Plasticity}

The above experiments were performed under standardized environmental conditions to detect genetically based differences in ontogenetic determinants between lake and stream stickleback. However, to explore phenotypic plasticity in life history, a field experiment was needed. Our approach was to release juvenile lake stickleback produced in the laboratory into stream enclosures, and to track growth and the propensity to mature until the beginning of the subsequent reproductive season. Our expectation was that if the observed life history divergence in the wild is primarily genetically based, lake fish should maintain their typical life history phenotype (i.e., large size and delayed maturation) even when developing in stream habitats. Conversely, a strong phenotypically plastic component to life history divergence would cause transplanted lake fish to express the typical stream phenotype (i.e., reproduction at small size after one year). We note that ideally, this experiment would have included reciprocally transplanting stream fish to lake enclosures. However, technical constraints and difficulties in maintaining an adequate pelagic foraging environment within lake enclosures over a long time period (e.g., avoiding the establishment of zoobenthos on enclosure walls) precluded transplants in this direction.

Our field experiment involved nine total enclosures, three of which were constructed near each of our three stream sampling sites (details given in Table S1). Trapping confirmed that stickleback occurred naturally at each site. The enclosures were built by fitting perforated metal plates (4 $\mathrm{mm}$ diameter holes; $58 \%$ passage) vertically in the stream bed and shore, enclosing stream segments of $6 \mathrm{~m}$ length. The enclosures were oriented parallel to the shore, such as to reach approximately $1 \mathrm{~m}$ from the water's edge into the streams (Suppl. Fig. S1). The bottom and shore area within the enclosures was natural, and to minimize disturbance, construction work was performed 6 weeks prior to fish release (early June 2013). At this point, we also removed all adult and as many juvenile stickleback as possible by extensive minnow trapping and electrofishing. All enclosures were covered with a fine $40 \mathrm{~mm}$ nylon net to prevent bird predation.

To begin the experiment, two enclosures per site were stocked with (foreign) lake stickleback. As a control, the third enclosure at each site was stocked with individuals from the corresponding stream population (locals). The number of individuals released was 30 per enclosure, selected haphazardly from the laboratory stock tanks. Since the enclosures allowed the entrance of small juvenile stickleback from outside, we marked all experimental fish by clipping the second dorsal spine. The same treatment was also applied to 80 fish from the stock tanks that were subsequently maintained in the laboratory. This confirmed that clipping had no growth or survival consequences, and that this marking was irreversible and unambiguous. The releases occurred 8 weeks post-hatch (July 23, 2013). At that point, the mean mass of the released fish was $0.1 \mathrm{~g}$ (approximately $20 \mathrm{~mm}$ standard length) and did not differ among the populations (details not presented).

The enclosures were subjected to minnow trapping 10 , $14,18,23,31$, and 36 weeks after the release at the $\mathrm{HOH}$ site, and after 10, 14, 31, and 36 weeks at the $\mathrm{BOH}$ and NID sites. (To reduce work load, and because we expected little growth during winter, only one site was sampled during wintertime.) Unmarked resident fish that had entered the enclosures were always removed. Experimental fish were counted, weighed, and returned to their enclosure. During the last visit (March 27-April 2, 2014), all experimental fish were additionally inspected for reproductive status, euthanized, and stored in absolute ethanol. Individuals were considered reproductive if they displayed mature ovaries (females), or breeding dress (males), the former determined by dissection.

\section{Data Analysis}

To compare growth trajectories among the laboratory populations, we first averaged individual body mass measurements across the two replicate tanks, yielding a single data point per family and measurement round. Because hatching of the experimental families extended over 3 weeks but body mass measurements were generally performed within one-week windows irrespective of hatching date, combining body mass data across families within populations required standardizing for age. We did so by using locally weighted scatterplot smoothing (LOESS, a non-parametric regression; Cleveland 1979). Based on a first-order polynomial and a smoothing span of 0.75 , we predicted for each family the body mass at standardized ages defined by the latest-hatching families. We also used LOESS to visualize population-specific mean growth trajectories and their associated $95 \%$ confidence bands (implemented in the ggplot2 R package; Wickham 2009).

To examine if the lake and the NID populations differed in their propensity to mature at the endpoint of the experiment (i.e., after 45 days), we analyzed the individual incidence of maturation in a generalized linear model with binomial error structure and experimental round and population as predictors. Because females and males differ in their reproductive physiology, this analysis was performed separately for each sex. $P$ values of the model terms and their interaction was established through (non- 
parametric) permutation (Manly 2007). We here randomized the response over the predictors 9999 times and used the distribution of the $\mathrm{z}$ value of each model term across the iterations to derive $P$ values.

To test for differences in body mass among the enclosures at end of the field transplant experiment (week 36 post-release), we used a linear model with body mass as response and enclosure as predictor. Data from each site were analyzed separately. $P$ values were established by permutation as above, using the model's $F$ value as test statistic. We also tested for each site if the stream fish raised in the (control) enclosure differed in size from their wild local counterparts at the end of the experiment. $P$ values were again generated by permutation, using the difference in population means (wild minus enclosure) as test statistic. All analyses and graphing were performed in the $\mathrm{R}$ statistical environment ( $\mathrm{R}$ Development Core Team 2014). The raw data from the growth trajectory, the maturation size threshold, and the field experiment are provided as Supporting Online Material.

\section{Results}

\section{Life History Divergence in the Field}

The field specimens collected in 2013 for the present study displayed life histories fully consistent with those observed earlier (Moser et al. 2012): body mass at reproduction was roughly twice as high in Lake Constance fish as in stream fish (Fig. 1b). Similarly, the lake population displayed an average age at reproduction of 2.1 years (range 1-3 y), as opposed to 1.2 years in stream fish (range 1-2 y). Life history divergence between lake and stream stickleback in the Lake Constance basin is thus temporally stable.

\section{Laboratory Experiments}

Our first hypothesis, a genetically determined lower growth rate in lake fish compared to stream fish, was clearly refuted by the analysis of growth trajectories. Although growth trajectories diverged in the course of the experiment, with the NID population growing slower than the other two populations, this difference was in the direction opposed to our prediction (Fig. 3). If anything, the intrinsic growth rate is higher in lake than stream fish.

In the maturation size threshold experiment, mean body mass in the beginning of all experimental rounds was substantially below the mean mass at reproduction observed in any population in the wild (range across all combinations of population, sex, and experimental round: 0.63-1.28 g, Table S2; compare to Fig. 1b). Because body mass tended to increase from the first to the third experimental round (Table S2), we also observed an

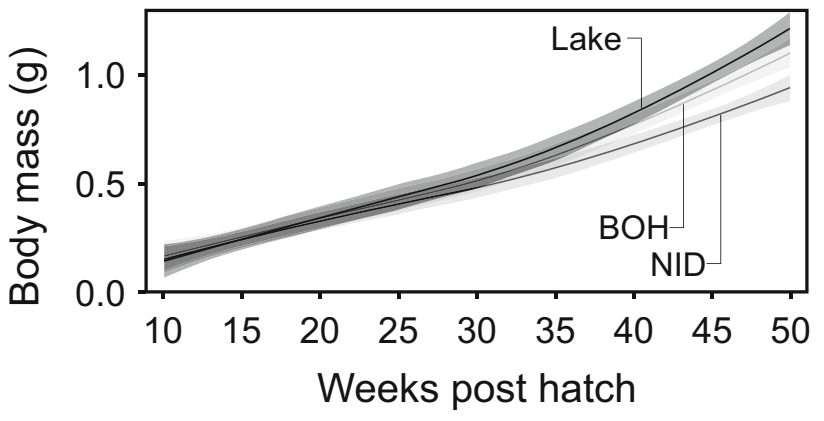

Fig. 3 Growth trajectories of the Lake Constance and two stream stickleback populations over 40 weeks under common garden conditions. Shown are mean trajectories across the replicate families within each population, along with the associated $95 \%$ confidence bands

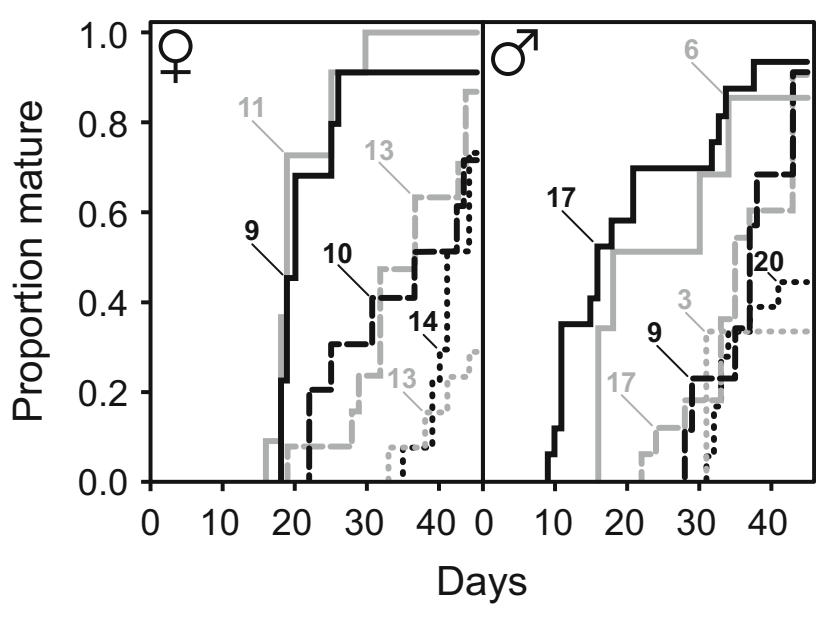

Fig. 4 The proportion of stickleback from the Lake Constance population (black) and a stream population (NID; gray) becoming reproductive during the maturation size threshold experiment, presented separately for females and males. The line styles designate the three experimental rounds (dotted, dashed, and solid for round 1, 2 , and 3 ) and the numbers indicate sample sizes

increasing propensity to mature over the experimental rounds (Fig. 4) (experimental round term, females: $P<0.001$; males: $P=0.16)$. Combining across the sexes and populations, the incidence of maturation rose from $46 \%$ (round 1) to $84 \%$ (round 2) and $93 \%$ (round 3 ).

The key outcome of the maturation size threshold experiment, however, was a lack of a difference in the propensity to mature between Lake Constance and NID stream in males (population term: $P=0.68$; round by population interaction: $P=0.33$ ), and a difference in the direction opposed to our prediction in females (population: $P=0.007$; interaction: $P=0.99$ ) (Fig. 4). However, the latter population difference should be taken with caution; due to slight differences in growth trajectories between the lake and NID populations (see above), lake fish were slightly larger than stream fish in the beginning of each 
experimental round. Combining across the sexes and experimental rounds, the overall propensity to mature was similar between lake and stream fish (75 and $72 \%)$. We further observed that across all experimental fish, individuals larger than $1 \mathrm{~g}$ at the start of an experimental round $(\mathrm{N}=41)$ always matured (Fig. S2). The minimum mass absolutely required for maturation could not be determined with confidence because the number of very small experimental individuals was insufficient, but is clearly below $0.5 \mathrm{~g}$ (Fig. S2).

Taken together, the maturation size threshold experiment demonstrates that both lake and stream fish can mature at body sizes much smaller than the typical size at reproduction seen in all populations in the wild. We find no support for the hypothesis that the switch to the reproductive stage is genetically determined to occur at a larger size in lake than in stream fish.

\section{Field Transplant Experiment}

At the NID field site, no individual in the two lake transplant enclosures survived longer than 10 weeks. Since we were interested in life history phenotypes expressed over longer time frames, this site had to be omitted from analysis.

At the $\mathrm{BOH}$ site, the transplanted lake stickleback were larger at the end of the experiment (week 36) than their stream conspecifics in the enclosures (Fig. 5; $P=0.005$ ). However, this result was based on a single lake enclosure because sometime between week 31 and 36, damage to the other lake enclosure allowed all experimental fish to escape. Analyzing the data from week 31, when both lake

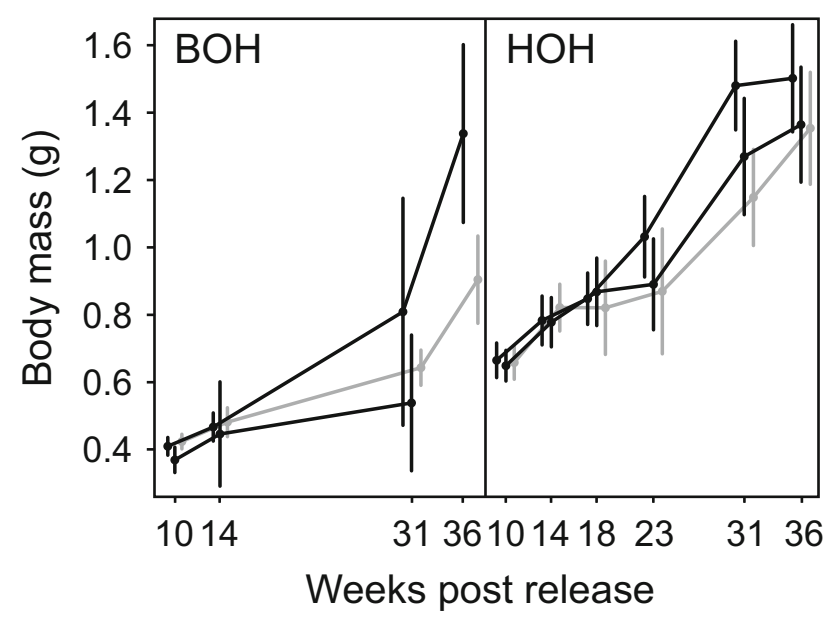

Fig. 5 Growth trajectories of stickleback exhibited under natural conditions in field enclosures at two study sites (the NID site was lost due to high mortality). Data from transplanted Lake Constance stickleback (two replicate enclosures per site) and local stream fish (a single control enclosure) are shown in black and gray. Error bars are $95 \%$ confidence intervals enclosures were still intact, we detected no body mass difference among the enclosures $(P=0.07)$. Similarly, there was no indication of body mass differences among the enclosures at the end of the experiment at the $\mathrm{HOH}$ site $(P=0.3)$.

At both the $\mathrm{BOH}$ and $\mathrm{HOH}$ site, control stream stickleback in the enclosures displayed slightly lower body mass at the end of the experiment than the corresponding resident stream population (Fig. 5, compare to Fig. 1b; BOH $P<0.001$, HOH $P=0.02$ ). However, this difference is not surprising because the enclosure experiment ended in March whereas the field populations (parental fish used to create the experimental fish) were sampled in May. We thus conclude that the growth trajectories displayed by stickleback within the enclosures are qualitatively similar to those of the wild local stream populations.

In all enclosures, stickleback started to mature toward the end of the experiment. At the $\mathrm{BOH}$ site, this was true for all individuals in the remaining lake enclosure $(\mathrm{N}=4)$, and for $64 \%$ of the individuals in the stream enclosure $(\mathrm{N}=17)$. At $\mathrm{HOH}, 28$ and $50 \%$ of the transplanted lake fish matured ( $\mathrm{N}=18$ and 22$)$, and a similar proportion of stream fish $(44 \%, \mathrm{~N}=27)$.

To summarize, the main insight from the field transplant experiment is that lake fish raised in replicated stream environments readily start reproducing after a single growing season. Further, they do so at body sizes typical of stream fish-that is, much smaller than the typical reproductive lake fish. Phenotypic plasticity thus causes a major life history shift within a single generation in Lake Constance stickleback.

\section{Discussion}

\section{The Mechanism of Life History Divergence Between Lake and Stream Stickleback}

The objective of this study was to combine multiple experiments to identify the mechanism(s) underlying life history divergence among stickleback populations in the Lake Constance basin. In this system, lake fish have been shown to reproduce at much larger size and later in life than neighboring stream populations (Lucek et al. 2012; Moser et al. 2012), a pattern here confirmed based on temporally independent samples.

Studying growth trajectories under controlled laboratory conditions revealed some degree of genetically based differentiation among the study populations, with Lake Constance fish displaying the fastest growth. This result agrees with a previous laboratory study based on a different lakestream population pairing from the Lake Constance basin that also suggested slightly faster growth in lake than 
stream fish (Lucek et al. 2012). The difference in growth among the populations might be due to divergence in the rate of food consumption, in the efficiency of food conversion, or both (Present and Conover 1992; Silverstein et al. 1999; Jonassen et al. 2000; Trudel et al. 2001). Tentative support for the former derives from the qualitative observation during our laboratory growth experiment that Lake Constance fish were bolder and foraged more actively than stream fish. However, formal experiments would be needed to quantify the relative contribution of foraging behavior and physiology to the genetic variation in growth, ideally including different food availability treatments to explore if stronger divergence is apparent when food is restricted.

Whatever the cause(s) for the different growth trajectories under laboratory conditions, these differences clearly cannot explain the variation in age and size at reproduction among the wild populations: first, the intrinsic differences in growth trajectories are too subtle to explain the large body size divergence within the Lake Constance basin. Second and most importantly, faster growth would make lake fish more likely than stream fish to attain the maturation size threshold within a single growing season, hence conflicting with the observation of delayed reproduction in Lake Constance stickleback relative to the stream populations (Fig. 2a).

Furthermore, our investigation of maturation size thresholds in the laboratory makes clear that both lake and stream genotypes can reproduce at body sizes well below the size of wild reproductive stream stickleback. The delayed reproduction of Lake Constance stickleback relative to their conspecifics in streams thus cannot be caused by the genetically based evolution of an elevated critical maturation size. Collectively, our laboratory experiments indicate that life history divergence within the Lake Constance basin primarily represents phenotypic plasticity. This view is confirmed by our field transplant experiment, demonstrating that Lake Constance fish developing in stream habitats display life history phenotypes similar to typical stream fish-i.e., maturity within one year at small size.

Which specific ontogenetic determinants (i.e., growth rate, the maturation size threshold, or both) and ecological factors conspire to generate this life history plasticity cannot be inferred directly from our experiments. However, additional evidence allows us to propose that differential growth rates resulting from different resource availabilities between the lake and stream habitat are responsible for life history plasticity, consistent with model A in Fig. 2. Support for this view is offered by quantitative data on the temporal development of zooplankton availability in Lake Constance, revealing a single zooplankton abundance peak from late spring to early summer (Sommer
1985). During the rest of the year, zooplankton abundance is low. Juvenile lake stickleback thus appear to be born into a habitat where resource availability is rapidly declining, and they additionally have to compete for food with larger individuals from previous age cohorts. This suggests that compared to its tributaries, Lake Constance is a relatively poor foraging environment over most of the year, generally supporting a growth rate too low to reach critical maturation size within one growing season. (Nevertheless, the large body size reached by Lake Constance fish after two years suggests that during peak zooplankton abundance, growth rate is high.) The demonstration of a large proportion of very small (i.e., below $0.5 \mathrm{~g}$ ) non-reproductive stickleback during the breeding season in the Lake Constance population would provide definitive evidence of this scenario.

By contrast, stomach content analysis in a supplementary sample of stream stickleback from the NID population taken in late December 2014 indicates that in streams, prey resources are abundant throughout the year (Table S3). Also, in this habitat, competitors from previous age cohorts are rare (Moser et al. 2012). Accordingly, our field transplant experiment demonstrates that stream habitats allow for substantial growth over fall and winter (i.e., between the weeks 14 and 31 in Fig. 5). Streams thus appear to be relatively profitable habitats generally allowing stickleback to attain critical maturation size within one growing season. If this proves generally true, it offers a plausible explanation for the convergence in body size among stream stickleback populations observed at a worldwide scale (see Fig. 4 in Moser et al. 2012).

\section{Implications}

Overall, we provide strong evidence that life history divergence between lake and stream stickleback in the Lake Constance basin is the outcome of resource-mediated phenotypic plasticity in growth trajectories between the habitats. Our study challenges the suggestion that divergence in size at reproduction within the Lake Constance basin is due to the genetically based adaptive evolution of faster growth in lake fish (Lucek et al. 2012). The different conclusions emerging from Lucek et al. (2012) and the present work emphasize three important aspects: first, understanding life history evolution requires explicitly considering the mechanisms through which growth and reproductive function are coordinated (Day and Rowe 2002; Berner and Blanckenhorn 2007). In our stickleback system, this concerns the interplay between growth rate, the maturation size threshold, and the photoperiodic maturation cue (Baggerman 1985). Considering these determinants jointly makes clear that large size at 
reproduction in Lake Constance fish cannot readily be attributed to faster growth, but-perhaps counterintuitively-to slower growth early in life.

Second, caution is warranted when interpreting phenotypic divergence in natural populations based on laboratory experiments alone. While stickleback in the Lake Constance basin certainly exhibit some genetically based differences in growth rate detectable in the laboratory, these differences are overwhelmed by phenotypic plasticity in the wild.

Third, adaptationist interpretations of phenotypic divergence in nature should be made with caution. We recognize that the slightly elevated growth rate of lake fish under laboratory conditions might represent a genetically based adaptation partly compensating for the generally low resource availability in that habitat, and thus might provide an example of countergradient variation (Conover and Schultz 1995). Nevertheless, most of the life history divergence between our lake and stream populations is plastic, and whether this plasticity is adaptive or simply an unavoidable response of the ontogenetic machinery to ecological differences between the habitats (Berner and Blanckenhorn 2007; Fitzpatrick 2012) requires experimental investigation.

Finally, our study has important potential implications for speciation. Lake Constance stickleback enter tributaries for reproduction. The breeding grounds of lake and stream fish thus certainly overlap (at least in streams where physical dispersal barriers are absent), providing the opportunity for lake-stream gene flow. However, phenotypic plasticity maintains a prominent body size difference between the habitats, and body size to govern reproductive interactions in several population within this species (Dufresne et al. 1990; Nagel and Schluter 1998; Ishikawa and Mori 2000; Albert 2005; Boughman et al. 2005; McKinnon et al. 2004, 2012; Conte and Schluter 2013). We therefore propose that in lake and stream stickleback in the Lake Constance basin, the colonization of ecologically distinct habitats immediately promotes reproductive isolation via sexual barriers. Experimentally evaluating the role of body size in sexual isolation in this system thus emerges as on obvious avenue for future research.

Acknowledgments This work benefited greatly from many people who aided fieldwork, helped raise fish, and provided access to the study populations: Jon Bättig, Dieter Dziuba, Anja Frey, Reinhard Gartner, Friedhelm Glönkler, Manfred Gutsche, Roman Kistler, Patricia Koch, Manuel Konrad, Anton Krüger, Alban Lunardon, Milo Moser, Marcel Nater, Peter Nater, Reinhard Nitzinger, Catherine Peichel, Sabine Person, Marius Roesti, Attila Rüegg, Christian Vögeli, and Markus Zellweger. Walter Salzburger and Patricia Holm kindly shared lab resources and infrastructure. Marco Colombo and two reviewers provided valuable suggestions on the manuscript. Financial support was provided by the Swiss National Science Foundation (grant 31003A $146208 / 1$ to DB) and by the University of Basel.
Ethical standard All experiments comply with the current laws of Austria, Germany and Switzerland.

Conflict of interest The authors declare no conflict of interest.

\section{References}

Albert, A. Y. K. (2005). Mate choice, sexual imprinting, and speciation: A test of a one-allele isolating mechanism in sympatric sticklebacks. Evolution, 59(4), 927-931.

Arnegard, M. E., McGee, M. D., Matthews, B., Marchinko, K. B., Conte, G. L., et al. (2014). Genetics of ecological divergence during speciation. Nature, doi:10.1038/nature13301.

Baggerman, B. (1985). The roles of daily and annual biological rhythms in the photoperiodic regulation of the breeding season in the stickleback Gasterosteus aculeatus L. Behaviour, 93(1/4), $1-7$.

Bernardo, J. (1993). Determinants of maturation in animals. Trends in Ecology \& Evolution, 8(5), 166-173.

Berner, D., Adams, D. C., Grandchamp, A.-C., \& Hendry, A. P. (2008). Natural selection drives patterns of lake-stream divergence in stickleback foraging morphology. Journal of Evolutionary Biology, 21(6), 1653-1665.

Berner, D., \& Blanckenhorn, W. U. (2007). An ontogenetic perspective on the relationship between age and size at maturity. Functional Ecology, 21(3), 505-512.

Berner, D., Grandchamp, A.-C., \& Hendry, A. P. (2009). Variable progress toward ecological speciation in parapatry: Stickleback across eight lake-stream transitions. Evolution, 63(7), 1740-1753.

Berner, D., Kaeuffer, R., Grandchamp, A.-C., Raeymaekers, J. A. M., Räsänen, K., \& Hendry, A. P. (2011). Quantitative genetic inheritance of morphological divergence in a lake-stream stickleback ecotype pair: Implications for reproductive isolation. Journal of Evolutionary Biology, 24(9), 1975-1983.

Berner, D., Roesti, M., Hendry, A. P., \& Salzburger, W. (2010). Constraints on speciation suggested by comparing lake-stream stickleback divergence across two continents. Molecular Ecology, 19(22), 4963-4978.

Boughman, J. W., Rundle, H. D., \& Schluter, D. (2005). Parallel evolution of sexual isolation in sticklebacks. Evolution, 59(2), 361-373.

Chung, H., Loehlin, D. W., Dufour, H. D., Vaccarro, K., Millar, J. G., \& Carroll, S. B. (2014). A single gene affects both ecological divergence and mate choice in Drosophila. Science, 343(6175), $1148-1151$.

Cleveland, W. S. (1979). Robust locally weighted regression and smoothing scatterplots. Journal of the American Statistical Association, 74(368), 829-836.

Conover, D. O., \& Schultz, E. T. (1995). Phenotypic similarity and the evolutionary significance of countergradient variation. Trends in Ecology \& Evolution, 10(6), 248-252.

Conte, G. L., \& Schluter, D. (2013). Experimental confirmation that size determines mate preference phenotype matching in a stickleback species pair. Evolution, 67(5), 1477-1484.

Coyne, J. A., \& Orr, H. A. (2004). Speciation. Sunderland: Sinauer Associates Inc.

Craig-Bennett, A. (1931). The reproductive cycle of the three-spined stickleback, Gasterosteus aculeatus, Linn. Philosophical Transactions of the Royal Society B. doi:10.1098/rstb.1931.0005.

Crispo, E. (2008). Modifying effects of phenotypic plasticity on interactions among natural selection, adaptation and gene flow. Journal of Evolutionary Biology, 21(6), 1460-1469. 
Day, T., \& Rowe, L. (2002). Developmental thresholds and the evolution of reaction norms for age and size at life-history transitions. The American Naturalist, 159(4), 338-350.

Dufresne, F., FitzGerald, G. J., \& Lachance, S. (1990). Age and sizerelated differences in reproductive success and reproductive costs in threespine sticklebacks (Gasterosteus aculeatus). Behavioral Ecology, 1(2), 140-147.

Fitzpatrick, B. M. (2012). Underappreciated consequences of phenotypic plasticity for ecological speciation. International Journal of Ecology, doi:10.1155/2012/256017.

Fuller, R. C. (2008). Genetic incompatibilities in killifish and the role of environment. Evolution, 62(12), 3056-3068.

Gavrilets, S., Vose, A., Barluenga, M., Salzburger, W., \& Meyer, A. (2007). Case studies and mathematical models of ecological speciation. 1. Cichlids in a crater lake. Molecular Ecology, 16(14), 2893-2909.

Hatfield, T. (1997). Genetic divergence in adaptive characters between sympatric species of stickleback. The American Naturalist, 149(6), 1009-1029.

Hawthorne, D. J., \& Via, S. (2001). Genetic linkage of ecological specialization and reproductive isolation in pea aphids. Nature, 412(6850), 904-907.

Hendry, A. P. (2004). Selection against migrants contributes to the rapid evolution of ecologically dependent reproductive isolation. Evolutionary Ecology Research, 6(8), 1219-1236.

Hendry, A. P., Nosil, P., \& Rieseberg, L. H. (2007). The speed of ecological speciation. Functional Ecology, 21(3), 455-464.

Hendry, A. P., \& Taylor, E. B. (2004). How much of the variation in adaptive divergence can be explained by gene flow? An evaluation using lake-stream stickleback pairs. Evolution, 58(10), 2319-2331.

Ishikawa, M., \& Mori, S. (2000). Mating success and male courtship behaviors in three populations of the threespine stickleback. Behaviour, 137(7-8), 1065-1080.

Jonassen, T. M., Imsland, A. K., Fitzgerald, R., Bonga, W., Ham, V., Nævdal, G., et al. (2000). Geographic variation in growth and food conversion efficiency of juvenile Atlantic halibut related to latitude. Journal of Fish Biology, 56(2), 279-294.

Kitano, J., Ross, J. A., Mori, S., Kume, M., Jones, F. C., Chan, Y. F., et al. (2009). A role for a neo-sex chromosome in stickleback speciation. Nature, 461(7267), 1079-1083.

Kozak, G. M., Head, M. L., \& Boughman, J. W. (2011). Sexual imprinting on ecologically divergent traits leads to sexual isolation in sticklebacks. Proceedings of the Royal Society B: Biological Sciences, 278(1718), 2604-2610.

Lavin, P. A., \& McPhail, J. D. (1993). Parapatric lake and stream sticklebacks on northern Vancouver Island: Disjunct distribution or parallel evolution? Canadian Journal of Zoology, 71(1), $11-17$.

Levin, D. A. (2009). Flowering-time plasticity facilitates niche shifts in adjacent populations. New Phytologist, 183(3), 661-666.

Lexer, C., Lai, Z., \& Rieseberg, L. H. (2004). Candidate gene polymorphisms associated with salt tolerance in wild sunflower hybrids: Implications for the origin of Helianthus paradoxus, a diploid hybrid species. New Phytologist, 161(1), 225-233.

Lowry, D. B., \& Willis, J. H. (2010). A widespread chromosomal inversion polymorphism contributes to a major life-history transition, local adaptation, and reproductive isolation. PLoS Biology,. doi:10.1371/journal.pbio.1000500.

Lucek, K., Roy, D., Bezault, E., Sivasundar, A., \& Seehausen, O. (2010). Hybridization between distant lineages increases adaptive variation during a biological invasion: Stickleback in Switzerland. Molecular Ecology, 19(18), 3995-4011.

Lucek, K., Sivasundar, A., \& Seehausen, O. (2012). Evidence of adaptive evolutionary divergence during biological invasion. PLOS ONE,. doi:10.1371/journal.pone.0049377.
Maan, M. E., \& Seehausen, O. (2011). Ecology, sexual selection and speciation. Ecology Letters, 14(6), 591-602.

Manly, B. F. J. (2007). Randomization, bootstrap and Monte Carlo methods in biology. Boca Raton: Chapman \& Hall.

McKinnon, J. S., Hamele, N., Frey, N., Chou, J., McAleavey, L., Greene, J., et al. (2012). Male choice in the stream-anadromous stickleback complex. PLOS ONE, doi:10.1371/journal.pone. 0037951.

McKinnon, J. S., Mori, S., Blackman, B. K., David, L., Kingsley, D. M., Jamieson, L., et al. (2004). Evidence for ecology's role in speciation. Nature, doi:10.1038/nature02556.

Moser, D., Roesti, M., \& Berner, D. (2012). Repeated lake-stream divergence in stickleback life history within a Central European lake basin. PLoS ONE, doi:10.1371/journal.pone.0050620.

Nagel, L., \& Schluter, D. (1998). Body size, natural selection, and speciation in sticklebacks. Evolution, 52(1), 209-218.

Nijhout, H. F. (2003). The control of body size in insects. Developmental Biology, 261(2003), 1-9.

Nosil, P. (2012). Ecological speciation. Oxford: Oxford University.

Nosil, P., Vines, T. H., \& Funk, D. J. (2005). Reproductive isolation caused by natural selection against immigrants from divergent habitats. Evolution, 59(4), 705-719.

Payne, R. B., Payne, L. L., Woods, J. L., \& Sorenson, M. D. (2000). Imprinting and the origin of parasite-host species associations in brood-parasitic indigobirds. Vidua chalybeata. Animal Behaviour, 59(1), 69-81.

Present, T. M. C., \& Conover, D. O. (1992). Physiological basis of latitudinal growth differences in Menidia menidia: Variation in consumption or efficiency? Functional Ecology, 6(1), 23-31.

R Development Core Team. (2014). R: A language and environment for statistical computing. Vienna: R Foundation for Statistical Computing.

Raeymaekers, J. A. M., Boisjoly, M., Delaire, L., Berner, D., Räsänen, K., \& Hendry, A. P. (2010). Testing for mating isolation between ecotypes: Laboratory experiments with lake, stream and hybrid stickleback. Journal of Evolutionary Biology, . doi:10.1111/j.1420-9101.2010.02133.x.

Ravinet, M., Prodoehl, P. A., \& Harrod, C. (2013). Parallel and nonparallel ecological, morphological and genetic divergence in lake-stream stickleback from a single catchment. Journal of Evolutionary Biology, 26(1), 186-204.

Rego, C., Santos, M., \& Matos, M. (2007). Quantitative genetics of speciation: Additive and non-additive genetic differentiation between Drosophila madeirensis and Drosophila subobscura. Genetica, 131(2), 167-174.

Reimchen, T. E., Stinson, E. M., \& Nelson, J. S. (1985). Multivariate differentiation of parapatric and allopatric populations of threespine stickleback in the Sangan River watershed, Queen Charlotte Islands. Canadian Journal of Zoology, 63, 2944-2951.

Rice, W. R. (1987). Speciation via habitat specialization: The evolution of reproductive isolation as a correlated character. Evolutionary Ecology, 1(4), 301-314.

Ritchie, M. G. (2007). Sexual selection and speciation. Annual Review of Ecology Evolution and Systematics, 38, 79-102.

Rogers, S. M., \& Bernatchez, L. (2006). The genetic basis of intrinsic and extrinsic post-zygotic reproductive isolation jointly promoting speciation in the lake whitefish complex (Coregonus clupeaformis). Journal of Evolutionary Biology, 19(6), 1979-1994.

Rundle, H. D., \& Nosil, P. (2005). Ecological speciation. Ecology Letters, 8(3), 336-352.

Schluter, D. (2000). The ecology of adaptive radiation. Oxford: Oxford University Press.

Shimada, Y., Shikano, T., Kuparinen, A., Gonda, A., Leinonen, T., \& Merilä, J. (2011). Quantitative genetics of body size and timing of maturation in two nine-spined stickleback (Pungitius 
pungitius) populations. PLOS ONE,. doi:10.1371/journal.pone. 0028859.

Silverstein, J. T., Wolters, W. R., \& Holland, M. (1999). Evidence of differences in growth and food intake regulation in different genetic strains of channel catfish. Journal of Fish Biology, 54(3), 607-615.

Smith, G., Fang, Y., Liu, X., Kenny, J., Cossins, A. R., de Oliveira, C. C., et al. (2013). Transcriptome-wide expression variation associated with environmental plasticity and mating success in cactophilic Drosophila mojavensis. Evolution, 67(7), 1950-1963.

Sobel, J. M., Chen, G. F., Watt, L. R., \& Schemske, D. W. (2010). The biology of speciation. Evolution, 64(2), 295-315.

Sommer, U. (1985). Seasonal succession of phytoplankton in Lake Constance. BioScience, 35(6), 351-357.

Streisfeld, M. A., Young, W. N., \& Sobel, J. M. (2013). Divergent selection drives genetic differentiation in an R2R3-MYB transcription factor that contributes to incipient speciation in Mimulus aurantiacus. PLoS Genetics,. doi:10.1371/journal. pgen.1003385.
Terai, Y., Seehausen, O., Sasaki, T., Takahashi, K., Mizoiri, S., Sugawara, T., et al. (2006). Divergent selection on opsins drives incipient speciation in Lake Victoria cichlids. PLoS Biology,. doi:10.1371/journal.pbio.0040433.

Thibert-Plante, X., \& Gavrilets, S. (2013). Evolution of mate choice and the so-called magic traits in ecological speciation. Ecology Letters, 16(8), 1004-1013.

Thibert-Plante, X., \& Hendry, A. P. (2009). Five questions on ecological speciation addressed with individual-based simulations. Journal of Evolutionary Biology, 22(1), 109-123.

Thibert-Plante, X., \& Hendry, A. P. (2011). The consequences of phenotypic plasticity for ecological speciation. Journal of Evolutionary Biology, 24(2), 326-342.

Trudel, M., Tremblay, A., Schetagne, R., \& Rasmussen, J. (2001). Why are dwarf fish so small? An energetic analysis of polymorphism in lake whitefish (Coregonus clupeaformis). Canadian Journal of Fisheries and Aquatic Sciences, 58(2), 394-405.

Wickham, H. (2009). ggplot2: Elegant graphics for data analysis. New York: Springer. 